\title{
MULTISENSOR MICROWAVE SEA-ICE CLASSIFICATION
}

\author{
Quimn P. Remund \\ Microwave Earth Remote Sensing Laboratory \\ Brigham Young University, \\ Provo, Utah
}

\begin{abstract}
Understanding the polar ice regimes is fundamental to the understanding of global climate and other geophysical processes. Sea ice characteristics can be grouped into a number of general sea ice classes. Multisensor data from NSCAT, ERS-2, and SSM/ is reconstructed into enhanced resolution imagery for use in ice type classification. The resulting 12 -dimensional data set is linearly transformed through principal component analysis to reduce data dimensionality and noise levels. An iterative statistical data segmentation algorithm is developed using maximum a prasteriori techniques. The conditional probability distributions of observed vectors given the ice type are assumed to be Gaussian. The cluster centroids, covariance matrices, and $a$ priori distributions are estimated from the classification of a previous temporal image set. An initial classification is produced using centroid training data and a weighted nearest neighbor classifier. Though validation is limited, the algorithm results in an ice classification which is subjectively superior to a conventional $k$-means approach.
\end{abstract}

\section{Introduction}

Because the polar regions of the earth play a critical role in the global climate, the remote sensing community has had a keen interest in polar sea ice characteristics. Polar sea ice influences heat transfer between the warmer ocean and cooler atmosphere. In this process, ice thickness and density is particularly important. The extent and surface characteristics of sea ice affects the global radiation budget by regulating the amount of solar radiation reflected back out into space. In addition, these regions infiuence the planetary water exchange cycle as well as local biota distributions. Sea ice is also considered a sensitive indicator of long term global climate change [1]. Hence, an accurate knowledge of important surface characteristics of sea ice is a valuable tool in understanding these geophysical processes.

Microwave remote sensing provides an excellent means for monitoring polar sea ice. Both active and passive microwave signatures are much less sensitive to atmospheric distortions than measurements collected at optical frequencies. This is particularly valuable in the Arctic and Antarctic where extensive cloud cover is common. Many research studies have shown that microwave signatures of sea ice are sensitive to surface parameters. In addition, microwave sensors do not require solar illumination to collect measurements of the surface. However, these benefits often come at the expense of spatial resolution.

Fundamental sea ice characteristics can be grouped into a number of general sea ice classes or types. Several studies have been pursued to classify ice type from observed microwave signatures. A single-band classifier using $33.6 \mathrm{GHz}$ passive high-resolution aerial measurements was used on Beaufort Sea data [2]. Kwok et al. developed a method for classifying high-resolution ERS1 SAR imagery using ancillary data from meteorological databases [3]. The primary strengths of these approaches lie in the high spatial resolution capability of the instruments. Consequently, image pixels are much less likely to contain a mixture of ice types. Lower resolution techniques have also been proposed. Wensnahan et al. proposed a classification method using passive radiometer data [4] to estimate the concentrations of first-year, multiyear, and thin ice in the Arctic. In [5], a classifier was developed that uses only ERS-1 scatterometer data. Finally, a neural network classifier for sea ice type is given in [6]. These studies are representative of the some of the different work that has been done in microwave sea ice classification.

This paper presents a mulitsensor sea ice classification approach which uses multispectral, dual-polarization data collected from both active and passive spaceborne instruments for the segmentation of Antarctic data. The proposed algorithm uses principal component analysis methods to reduce the dimensionality of the data set and reduce noise effects. An iterative classification scheme is introduced using maximum a poskerioni techniques.

\section{Background}

The proposed ice classification scheme uses data from several different spaceborne instruments. This section provides a brief background of each of the data collecting instruments and the corresponding ice type signatures. In addition, the methods for image reconstruction and ice masking are described.

\section{Spaceborne Microwave Sensors}

Data from three different sensors are used in the classification approach which follows. The sensors are chosen 
for their temporal simultaneity of measurement collection during the target time frame of September-October 1996. In addition, all of the selected instruments have largescale coverage capability. The first data set comes from the NASA scatterometer (NSCAT) that flew from August 1996 to June 1997. NSCAT is a dual-polarization, dualswath, fan-beam scatterometer that collects Ku-Band measurements at multiple azimuth and incidence angles.

The second sensor is the active microwave instrument (AMI) aboard the European remote sensing satellite (ERS2). One mode of operation of the AMI is scatterometer mode which measures the vv-pol normalized radar cross section $\left(\sigma^{\circ}\right)$ at several azimuth and incidence angles. Similar to NSCAT, ERS-2 is a fan-beam wind scatterometer but it has only a single side-looking swath.

Finally, passive radiometer data is used in concert with the active scatterometer data to produce a merged data set. The special sensor microwave imager (SSM/I) aboard the Defense Meteorological Satellite Program series of satellites is a seven channel, four frequency radiometer. The channels are h- and v-pol at 19.35, 37.0, and 85.5 $\mathrm{GHz}$ and v-pol at $22.235 \mathrm{GHz}$. Brightness temperature $\left(T_{B}\right)$ measurements are collected from each channel. The $3 \mathrm{~dB}$ antenna fooprints range from about $15-70 \mathrm{~km}$ in the along-track direction and $13-43 \mathrm{~km}$ in the cross-track direction.

\section{Image Reconstruction}

While the inherent resolutions of the various instruments are sufficient for the stady of large-scale phenomena such as surface winds or atmospheric parameters, they can be too low for use in some studies. In an effort to ameliorate this problem and to place the data on compatible grids, the scatterometer image reconstruction (SIR) algorithm is used to enhance the spatial resolution $[7,8]$. SIR is an iterative block multiplicative algebraic reconstruction technique that increases the resolution of reconstructed imagery through the use of multiple passes of the satellite.

For scatterometers, $\sigma^{\circ}$ (in $\mathrm{dB}$ ) has a nearly linear incidence angle dependence over a limited range of incidence angles, $\theta \in\left[20^{\circ}, 55^{\circ}\right]$ given by

$$
\sigma^{\circ}(\mathrm{d} B \mathrm{~B})=A+B\left(\theta-40^{5}\right)
$$

where $A$ is $r^{\circ}$ normalized to $40^{\circ}$ incidence and $B$ is the incidence angle dependence of $\sigma^{\circ}$. SIR creates images of both $A$ and $B$ for scatterometers. NSCAT images are reconstructed on a $4.45 \times 4.45 \mathrm{~km}$ grid with an effective resolution on the order of 8-10 km. ERS-2 images are generated on a $8.9 \times 8.9 \mathrm{~km}$ grid with an effective resolution of $20-25 \mathrm{~km}$.

A univariate version of SIR can be applied to radiometer data such as SSM/I [8]. The lower side lobes of SSM/I make resolution enhancement more difficult. However, a clear improvement in resolution is observed in the reconstructed imagery. That is, surface features are moxe clearly defined in SIR imagery than in nonenhanced images on the same grid. SSM// brightness temperature $\left(T_{g}\right)$ SIR images are reconstructed on a $8.9 \times 8.9 \mathrm{~km}$ grid for all channels except for $85 \mathrm{~V}$ and $85 \mathrm{H}$ which have a pixel spacing of $4.45 \times 4.45 \mathrm{~km}$.

All images are generated using six days of data with three days of overlap in consecutive images. Although NSCAI v-pol and SSM/I can achieve full coverage of the Antarctic ice pack in much less time, ERS-2 and NSCAT h-pol require the full six days. For consistency in pixel spacing between the different images, the $8.9 \mathrm{~km}$ images are interpolated to the $4.45 \mathrm{~km}$ grid. All parameter images are used in the classification except for the ERS-2 $B$ images which have relatively high noise levels. The final merged data set consists of 12-dimensions with three $A$, two $B$, and seven $T_{A}$ images.

\section{Ice Masking}

Open ocean pixels in the reconstructed imagery are masked out for two reasons. First, the sea ice classification algorithm presented below uses statistical preprocessing techniques which take advantage of the covariance structure of the data to reduce the dimensionality of the data space. Since ocean pixels have typically high covariance values in all of the active and passive signatures, undue weight would be given to ocean pixels in the new data space effectively reducing the classification potential. Second, a significant number of the image pixels are open ocean and the removal of these pixels reduces the size of the classification data set.

The ice extent mapping algorithm used in this study is described in [9]. The technique uses the NSCAT polarization ratio $\left(A_{v} / A_{h}\right)$ as well as the NSCAT v-pol incidence angle dependence of $\sigma^{\circ}\left(B_{v}\right)$ to discriminate between sea ice and ocean pixels. Linear and quadratic segmentation techniques are applied resulting in an estimate of sea ice extent. Since wind induced roughness of the sea surface causes ambiguities in the discrimination, a third parameter, the $\sigma^{a}$ estimate error standard deviation, is introduced. This metric is sensitive to temporal and azimuthal variations during the imaging period and is consequently quite high in regions of high winds. The resulting ice extent map, when compared with NASA-Team SSM/Iderived ice concentration maps, most closely corresponds with the $30 \%$ ice concentration edge.

\section{Ice Type Signatures}

Data collected by NSCAT, ERS-2, and SSM/I are used to segment the data into six general ice types or classes. While the following discussion is based on the general 
behavior of scattering and emission from sea ice, in silu measurement averages for the various ice classes can be found in [5] for C-Band scarterometer Antarctic data and [6] for Arctic SSM/I data.

The first ice type to be considered is smooth first-year (SFY) ice. This class is comprised of relatively young ice which has not been roughened by the motion of the ice pack. Ranging in thickness from $10 \mathrm{~cm}$ to $1 \mathrm{~m}$, smooth first-year ice is highly saline with a high density of brine pockets caught within the ice crystal lattice. The high salinity causes this ice type to be very lossy and thus dominated by surface scattering and emission at virtually all frequencies used in the study. The active signatures exhibit low $A$ and $B$ values due to the strong incidence angle of smooth surface scattering. $T_{R}$ measurements are expected to be relatively high.

Like smooth first-year ice, rough first-year (RFY) ice is very saline and lossy. Surface scattering and emission dominate the signatures. Motion within the ice pack causes extensive roughening of this ice type. In general, the rough surface scattering cause $A$ values to be higher than for smooth ice types and $B$ values to rise (ie, have less incidence angle dependence). While passive signatures are less sensitive to the difference in RFY and SFY ice classes, $T_{\boldsymbol{A}}$ values are radiometrically cooler for $R F Y$ when compared to SFY ice.

Perennial (PER) ice is another important Antarctic ice type. While multi-year ice is common in the Arctic, less Antarctic sea ice survives more than one summer's melt since the Antarctic continent limits the southern extent. Regardless, a small amount of perennial ice can be found and is included in the classification. Over time, brine drainage results in much lower salinity and hence lower loss in this ice type. This leads to greater penetration depths and volume contribution to scattering and emission. $A$ and $B$ values are typically higher than those for RFY ice while $T_{B}$ measurements are lower.

Another sea ice type to be considered in the classification is the iceberg class (IB) consisting of large fioes of fresh water ice that have calved off from an ice shelf. In the absence of surface melt, this ice class has very low loss resulting in a large contribution from volume scattering and emission especially at lower microwave frequencies. As a result, $A$ and $B$ are very high and $T_{B}$ values are very low compared to other ice classes. The volumetric contribution also causes a depolarization resulting in similar response for both $\mathrm{v}$ - and $\mathrm{h}$-pol measurements.

Pancake ice is also included in the classification effort This ice regime is normally found in the outer portions of the ice pack where wave action deforms thin ice into floes called pancakes. The high roughness of this type results in a signature that is very similar to perennial ice in both active and passive signatures.
The final ice type is the marginal ice zone (MIZ). This dynamic region of the ice pack consists of mixtures of ice and open water. The open water contribution drives $T_{B}$ down. Wind roughening of the ocean surface in these regions causes $A \mid$ and $B$ values to often be confused with other ice types.

One of the complicating factors in sea ice classification is the seasonal dependence of the ice type microwave signatures. The signatures are most distinct during the austral winter months when ice types exhibit negligible surface melt. Hence, the differences due to subsurface contributions are strong. When temperatures rise and water content increases, the scattering and emission from lower ice layers become increasingly masked and surface scattering mechanisms begin to dominate. This causes ice type clusters to drift in the 12-dimensional data space with some clusters merging together. For this reason, an effective classification technique must have the ability to adapt to changing signatures in order to maintain a maximal degree of accuracy.

\section{Multivariate Analysis}

As previously discussed, the classification data set consists of 12 parameters from which sea ice type is to be extracted. Additional preprocessing is performed on the data to maximize classification accuracy and minimize required computational effort. Since the parameters are measured in very different units, data fusion techniques are used to give equal weighting to all of the data In an effort to reduce the computational complexity and the noise levels, principal component analysis is implemented.

\section{Data Fusion}

The 12-dimensional data space consists of three basic types of data with differing units. The first data type, Al, is measured in $\mathrm{dB}$ with a typical range of -30.0 to $0.0 \mathrm{~dB}$. The incidence angle dependence of $\sigma^{\circ}$, given by $B$, contains $\mathrm{dB} / \mathrm{deg}$ values ranging from -0.4 to $-0.1 \mathrm{~dB} / \mathrm{deg}$. The last data type is $T_{B}$ measured in degrees Kelvin with sea ice values from $150 \mathrm{~K}$ to $290 \mathrm{~K}$ depending on frequency and polarization. Since each data type is quite different from the others, standardization is required to ensure that each data type is given appropriate weight in the classification. The standard approach is to shift and scale the data so that each parameter has zero mean and unit variance. However, this may remove some ice class information that exists in the mean parameter responses. In an effort to preserve the ice class dependent biases that exist in each data type, the following standardization technique is applied for a particular observation $x$

$$
x_{n}=\frac{\left(x-\mu_{t_{y p e}}\right)}{\sigma_{t y p e}}
$$


where $\psi_{\text {inype }}$ and $\sigma_{\text {twpe }}$ are the mean and standard deviations of all the data of a particular type (e.g., $A, B$, or $T_{R}$ data) and $2 a_{s}$ is the new standardized parameter value. Hence, all standardized $A, B$, and $T_{R}$ data have zero mean and unit. The resulting data resides in a 12dimensional unitless space in which each data type has similar variance.

\section{Principal Component Analysis}

The high dimensionality of the classification data set results in significant computational requirements. To reduce the number of required parameters, principal component analysis (PCA) is implemented. PCA is a powerful data analysis tool that effectively rotates the data space by projecting each observation onto a new orthonormal basis [10]. The resulting basis vectors are chosen such that the first spans the direction of maximum variance in the data Successive vectors are chosen to span the maximum variance not accounted for by previous vectors.

For the classification problem at hand, data vectors are composed of the 12 standardized values

$$
\vec{y}=\left[\begin{array}{llll}
y_{1} & y_{2} & \ldots & y_{12}
\end{array}\right]^{T}
$$

where the $y_{i}$ represent the standardized values of NSCAT, ERS-2, and SSM/I data values. PCA uses an eigenvectoreigenvalue decomposition of the data to construct the necessary orthonormal basis vectors. The eigen equation is given by

$$
\kappa \Gamma-\Gamma \Lambda
$$

where $K$ is the $12 \times 12$ covariance matrix of the standardized data, $\Gamma$ is a matrix containing the eigenvectors of $K$ along the columns (which form a basis for the original 12-dimensional space), and $\Lambda$ is a diagonal matrix with the eigenvalues of $K$ along the diagonal (which represent the variances spanned by each eigenvector). Once these are obtained, a $12 \times 1$ data vector $\vec{y}$ containing standardized parameters is transformed through projection onto the new basis

$$
\vec{z}=\Gamma^{T} \vec{y}
$$

The elements of $z$ are called the principal component scores [10].

The analysis technique is used on land/ice masked imagery to produce 12 principal component images composed of a combination of information contained in the original parameters. The pixel values in individual PCA images represent coefficients of the eigenvector associated with that principal component score. The size of the corresponding eigenvalues determine the variance and informational content of each of the images. For example, the PCA transformation was performed for the microwave data set during the imaging interval $1996 \mathrm{JD}$ 261-266. Figure 1 illustrates the resulting eigenvalue spectrum. Clearly, a majority of the data variance is contained

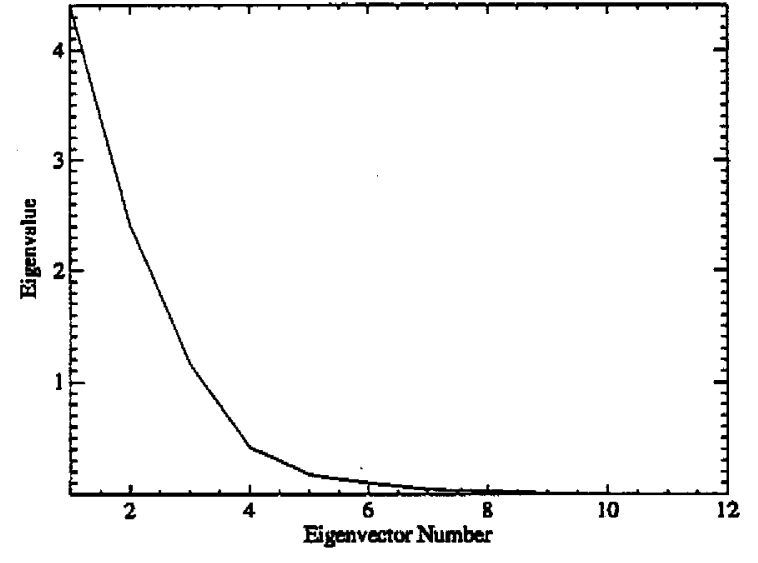

Figure 1: Eigenvalue spectrum for the principal component data rotation during the imaging interval of $1996 \mathrm{JD}$ 262-266. A large majority of the data variance is contained in the first few eigenvectors.

in the top few principal component images implying that lower PCA images can be neglected with minimal effect on the final classification. Wensnahan et al suggest keeping only PCA parameters whose variance is much larger than measurement uncertainty (converted into principal component space) [4]. Such a choice of eigenvectors allows information to be separated from noise. Indeed, the lower principal component images used in this study appear very noisy with image reconstruction artifacts dominating the features. Hence, by ignoring these eigenvectors, we eliminate undesirable noise as well as reduce data dimensionality.

Another method for the choosing of principal component images is to keep the top N PCA transformed images that account for some predetermined percentage of the total variance in the data. For this classification project, the eigenvectors that span $90 \%$ of the variance are kept for use in the data segmentation. Only three PCA images must be retained in the case of the sample data set representing $1996 \mathrm{JD}$ 261-266 Antarctic sea ice data.

PCA can be used not only to reduce the dimensionality and noise levels of the data, but to quantitatively assess the informational content of multisensor data. By observing the relative magnitudes of the elements of the first few eigenvectors, one can determine levels of informational content of the original parameters. For example, parameters with low corresponding elements for the top several eigenvectors likely have negligible informational content. Hence, these parameters can be eliminated without a major impact on the classification. 


\section{Iterative Algorithm Development}

Several techniques are available for classification of $\mathrm{N}$ dimensional data sets. A nearest neighbor approach is perhaps the simplest when centroids from training samples or electromagnetic models can be obtained. Iterative clustering algorithms such as k-means or ISODATA represent another methodology and search for natural clusters in the data. The task then remains to label the resulting clusters as different classes. In contrast, the proposed approach is a statistical classification scheme with the goal of maximizing the probability of correctly classifying sea ice type. This section presents the classification methodology through a development of an iterative maximum ma posteriori algorithm

\section{Statistical Classification}

The intrinsic value of statistical methods of classification stems from the ease of interpretation of results. That is, statistical classifiers attempt to maximize a probability measure given some level of knowledge of class distributions. Two primary branches have evolved in the field of statistical classification and estimation: maximum likelihood and Bayesian classification. The proposed algorithm focuses on the latter.

Bayesian methods represent one class of statistical approaches. This scheme requires the definition of a loss function which assigns a penalty for misclassifications. The Bayes solution is the one that minimizes the expected loss which is also called the Bayes risk. Under a uniform loss function this reduces to a maximum a pasterior (MAP) classifier. The MAP technique treats the ice type $C$ as a random variable and maximizes the probability of ice type given the observation vector $\vec{z}$

$$
C_{M A R}=\operatorname{argmax}_{a} p(C \mid \vec{z})=\operatorname{argmax}_{r} \frac{p(\vec{z} \mid C) p(C)}{p(\vec{z})}
$$

where $p(C)$ is the a prioni distribution. Since $p(\vec{z})$ is fixed for a particular observation, this reduces to

$$
C_{M A P}=\text { argmax, } p(z \mid C) p(C) .
$$

MAP classification has an advantage over maximum likelihood techniques since the probability of each class is included in the derivation ensuring that less likely ice types appear less frequently in the final classification. However, the a prioni distribution and the conditional distributions are required.

Under a Gaussian assumption, the conditional distributions are

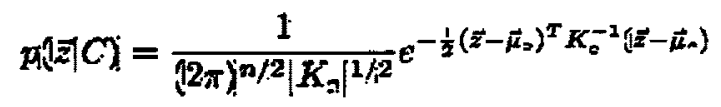

where $\vec{\mu}_{1}$ is the mean vector and $K_{a}$ is the covariance matrix of ice type $c$, respectively. Hence, the statist- cal structure of the data for each ice class is completely determined by the mean vectors and covariance matrices. Even if the Gaussian assumption is not entirely correct, it is considered to be an improvement over the simple isotropic distribution assumption inherent to a nearest neighbor classifier since the Gaussian model can account for covariance between separate principal component scores. This allows the classifier to use cluster shapes in addition to the centroids to segment the data.

With a Gaussian conditional density function, Eq. (7) can be simplified. After taking the natural $\log$ (a monotonic function) and a little mathematical manipulation we obtain

$$
\begin{aligned}
C_{\text {MAP }}= & \operatorname{argmax}_{\mathrm{c}} p(\vec{z} \mid C) p(C) \\
= & \operatorname{argmax} \mathbb{R}_{n}-\frac{1}{2}\left(\log \left|K_{\mathrm{s}}\right|+\right. \\
& \left.\left.\left(\vec{z}-\vec{\mu}_{c}\right)^{T} K_{c}^{-\mathrm{j}}\left(\vec{z}-\vec{\mu}_{c}\right)\right)+\log (p(C))\right] .
\end{aligned}
$$

This expression is used to segment the data.

\section{Iterative Approach}

In order to fully implement the MAP technique, the mean vectors $\vec{\mu}_{a}$ and covariance matrices $K_{\theta}$ of the individual ice type clusters are required along with the a prioni distribution $p . j C)$. While a rough estimate of the cluster centroids can be generated from small homogeneous training regions, it is more difficult to obtain reasonable estimates of the $K_{n}$ matrices. However, estimates can be obtained through an iterarive procedure, assuming that the statistical measures converge to the correct values.

Figure 2 illustrates the complete process for the classification of a time series of image data. The initial SIRderived images are first masked to remove all land and ocean pixels using the ice extent mapping procedure defined in an earlier section. The PCA linear transformation is then performed to rotate the coordinate space into ordered maximum variance axes. Next, the resulting 12 dimensional principal component space is truncated by choosing the top $N$ eigenvectors that span $90 \%$ of the data variance.

After the preprocessing, an iterative maximum a posierioni classifier is implemented. The first iteration uses the $\vec{\mu}_{c}, K_{n}$, and $p(C)$ statistical measures computed from the classification of the previous image set. Thus, the preceding classification is treated as a training set to obtain initial sea ice type cluster centroids, covariance matrices, and the a priori distribution. Due to the seasonal narure of cluster characteristics, these measures are likely erroneous. However, these represent a good initial starting point for the iterative procedure. After the first iteration, the statistical measures are updated using the new classification. These are then used in a new classification. The process iterates until predefined convergence criteria 


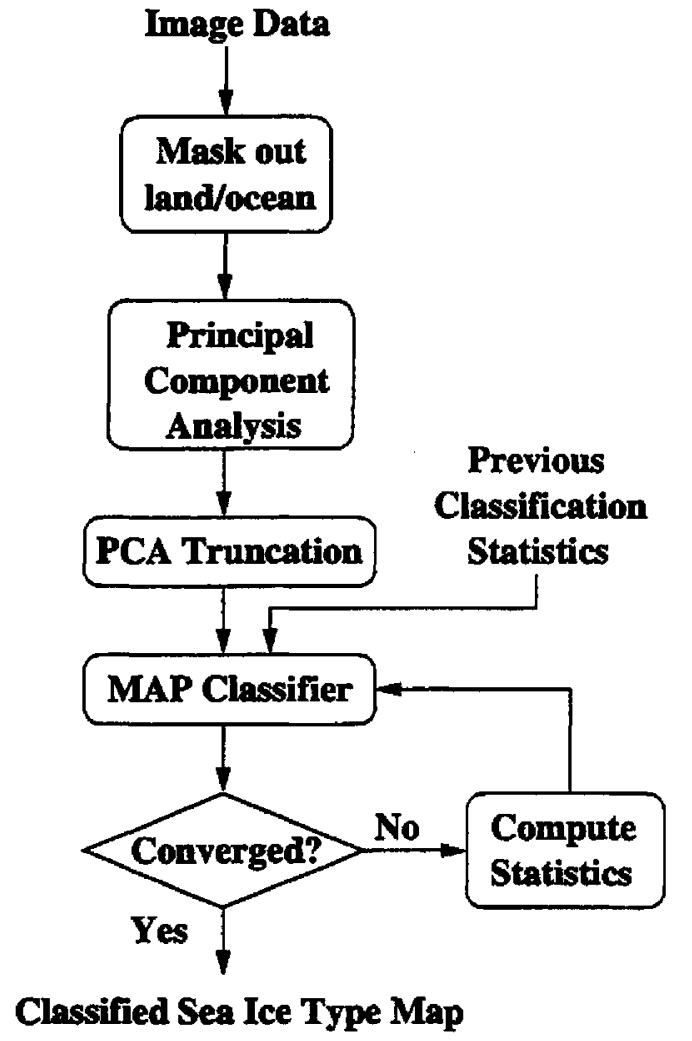

Figure 2: Flowchart depicting the iterative ice classification algorithm.

are met. The result is a classified image which maps the spatial extent of each sea ice type.

\section{Convergence Metrics}

Two metrics are used to determine algorithm convergence Since the Gaussian clusters are completely defined by the centroid vectors and covariance matrices, the appropriate norms are used to obtain scalar measures of individual cluster behavior. The Euclidean norm is used to measure the behavior of the cluster centroid vectors as a function of iteration. The matrix spectral norm of each covariance matrix $K_{n}$ is computed as a measure of the overall variance structure of each cluster. The spectral norm is equivalent to the square root of the maximum eigenvalue of $K_{a}^{T} K$. Convergence of both metrics for a particular cluster is a good indication that the cluster remains unchanged from one iteration to the next

\section{Algorithm Initialization}

The algorithm described above is a recursive method that uses the classification result from the previous imaging interval to compute the present sea ice type map. In order to obtain an initial classification result to start the process, the following procedure is used. Cluster centroid
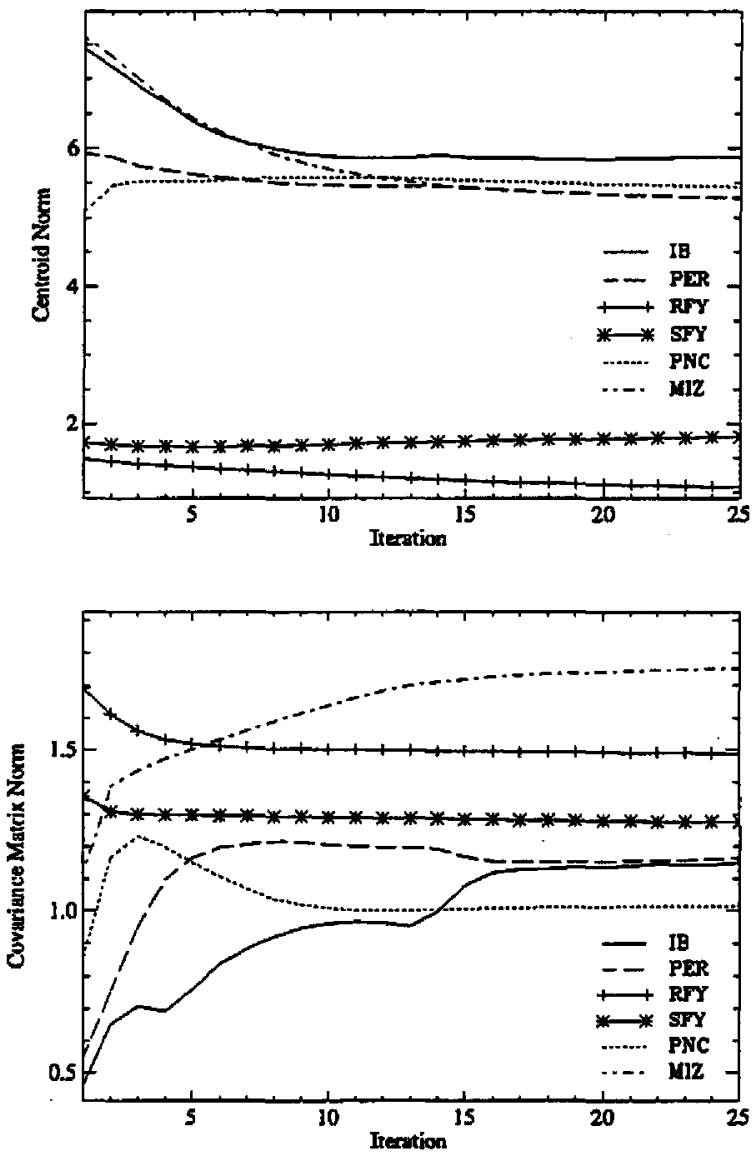

Figure 3: Maximum a postentiond classification cluster convergence metrics. (Top) The Euclidean norms of each ice type cluster as a function of iteration. (Bottom) The spectral norms of the covariance matrices.

vectors are estimated from small homogeneous training regions derived from a basic knowledge of sea ice type spatial behavior and expected microwave signatures. For the MAP method, the data is segmented with a weighted nearest neighbor technique in which the distances to each cluster are inversely weighted by an initial estimate of $p(C)$. While an accurate estimate of the a priond distribution is difficult to produce, an educated estimate can be made through a knowledge of sea ice type population in Antarctica. For example, a large majority of the Antarctic ice pack consists of various types of first-year ice. Other classes are much less prevalent. The nearest neighbor solution is used to compute the necessary statistics for the classifier and initiate the iterative algorithm.

Simulations of the algorithm are performed. The simulation data consists of four different two-dimensional Gaussian distributions with different mean vectors, covariance matrices, and cardinalities. The distributions are 


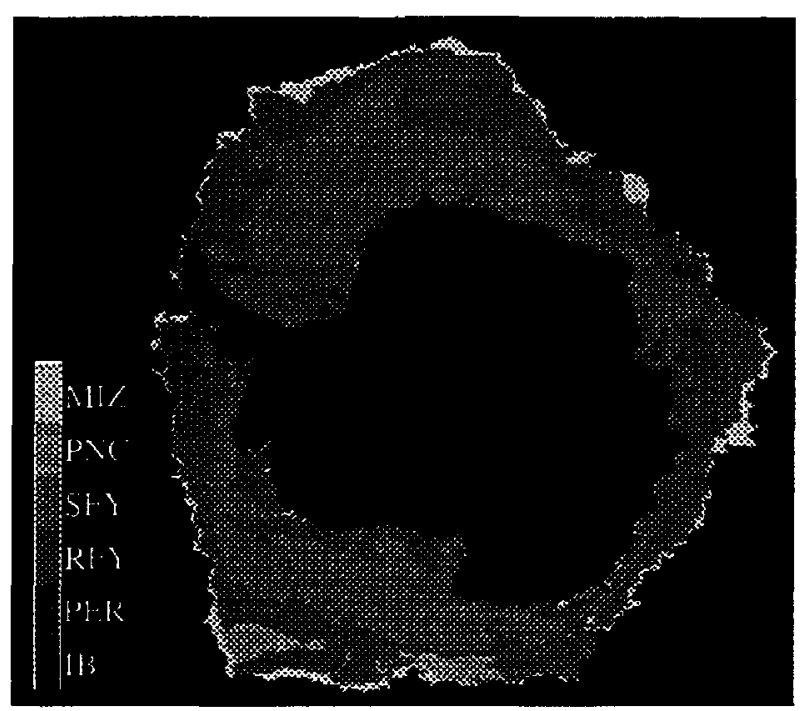

Figure 4: MAP sea ice classification of 1996 JD 261-266 Antarctic data.

chosen to have significant overlap to increase the classification difficulty. Simulation results indicate that the iterative algorithm converges to a solution that is very close to the actual MAP solution given two conditions: first, the individual cluster centroids must be relatively close to the actual centroids. In the simulations, this means that the centroid estimate merely has to be closer to its actual centroid than any of the others. Second, for MAP classification, the initial distribution estimate of $p(C)$ must be a reasonable estimate of the actual a prior $i$ distribution.

\section{Results}

The iterative algorithms are applied to the classification of Antarctic data during consecutive imaging periods in September and October of 1996. The algorithm is initiated with multisensor data from JD 261-266. As noted in the previous section, the nearest neighbor segmentation is required for the first image of the time series. Small homogeneous training regions are defined through a knowledge of sea ice dynamics and microwave signatures.

Figure 3 shows the convergence metrics as a function of iteration for the MAP classifications, respectively. After about 25 iterations, all metrics have converged relatively well. Some minimal centroid drift is still evident in the centroid norm trends. Only two of the MAP centroid norms move significantly indicating that the original centroids are reasonable estimates of the true values.

Figure 4 depicts the final sea ice type image. The result exhibits a reasonable spatial distribution of ice types. Several known icebergs are classified correctly. The largest concentration of perennial ice is found just off the tip of the Antarctic Peninsula. The ice here has survived the previous melt season by avoiding being swept out to sea

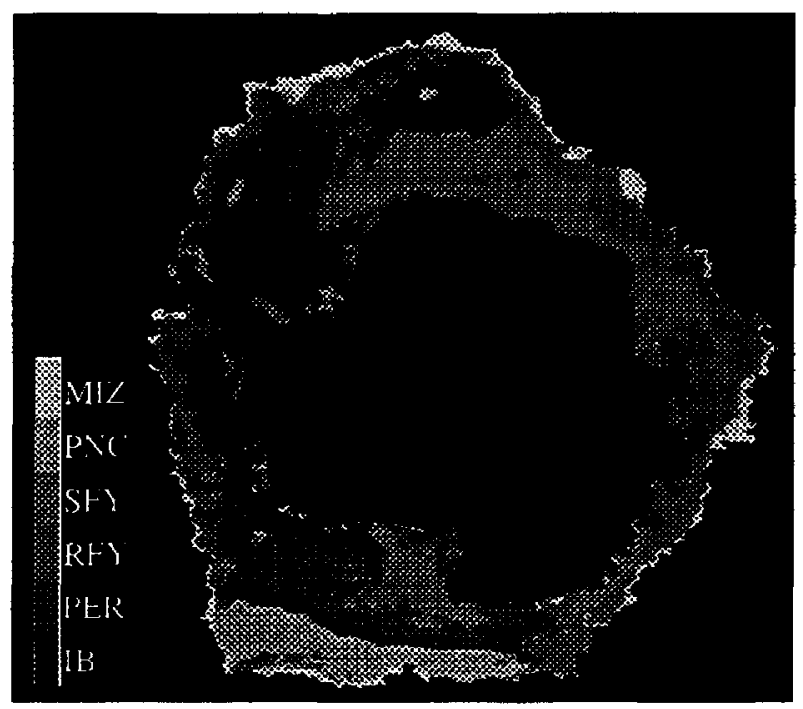

Figure 5: K-means clustering classification result of 1996 JD 261-266 Antarctic data.

by the Weddell Gyre. Rough first-year ice in the classification surrounds smooth first-year ice which is located primarily in the inner portion of the ice pack. This is consistent with the classification results in [5]. In addition, the marginal ice zone exists on the perimeter of the ice pack as expected.

Figure 5 shows classification image generated using another method for comparison. It was implemented using the same training data for initial cluster centroids. The image is the classification result of the standard $\mathrm{k}$ means clustering algorithm. The $\mathrm{k}$-means approach yields a solution that minimizes the within cluster sum of squared distances under the Euclidean distance metric. Since no regard is given to the probability of ice type, the k-means result overclassifies several ice types. For example, large extents of two rare ice types, icebergs and perennial ice, exist in the $\mathbf{k}$-means ice map indicating erroneous results.

An obvious error in the MAP classification is the RFY labeled tongue extending from the Ross Ice Shelf. The perimeter of the ice shelf is actually a region of new ice formation and divergence. Consequently, we conclude that the ice in this regime should have been identified as SFY. The source of the discrepancy is likely due to frost flower formation on the surface of smooth ice. Drinkwater and Crocker found that frost flower formation can yield microwave signatures that are similar to RFY ice [12]. The proposed classifier did not include a separate classification cluster for this ice type. A useful line of future research would include a study of the potential of segmenting frost flower covered ice from RFY ice. 


\section{Conclusions}

This study has demonstrated the utility of a multisensor, iterative maximum a posfleriori sea ice type classification algorithm for Antarctic sea ice. The use of data collected from multispectral, dual-polarization, active, and passive instruments increases the level of information that can be exploited in segmenting the data Through the use of principal component analysis, not only is the data dimensionality minimized, but the effects of noise and imaging artifacts are reduced. The resulting data set is classified in an iterative manner that utilizes MAP statistical techniques.

The iterative classification algorithm yields ice maps with spatial ice type distributions that are reasonable when general ice dynamics are considered. However, while the algorithm appears to function well, a more detailed validation study is needed. Unfortunately, validation data in the Antarctic is difficult to obtain. Though SAR data exists for continental Antarctica, sea ice SAR imagery during the period spanned by our multisensor data set is scarce. A useful future line of research would be to apply the algorithm to Arctic data where sea ice SAR imagery is much more abundant both spatially and temporally.

Several implications must be considered in a mediumscale classification such as the method presented in this study. First, the six day imaging period may introduce blurring in the images due to sea ice motion resulting in ambiguous signatures and misclassification. The limiting factor for this data set are the scatterometers which need more time to achieve full coverage of the Antarctic ice pack. In the future, similar algorithms may be applied with instruments with wider swaths such as the Seawinds scatterometer. Seawinds will reach full coverage in one to two days rather than six days and is scheduled for launch in 2000 . Also, the relatively low resolution, even in the reconstructed imagery, implies that some pixels may contain a mixture of ice types. Thus, the classification result for a particular pixel is considered the spatial and temporal average behavior of sea ice in that region. A promising line of future research is the extension of this algorithm from a hard to a fuzzy classifier. That is, for each pixel the concentration of each ice type may be estimated. It is conceivable that the MAP probabilities could be used to achieve this. However, a greater understanding of the effects of within-footprint mixtures on observed microwave signatures is needed.

\section{References}

[1] WF. Budd, "Antartic sea ice variations from satellite sensing in relation to climate," IEEE Trans. on Geosci. and Rem. Sens., vol. 15, pp. 417-426, 1975.

[2] D.T. Eppler, L.D. Farmer, A.W. Lobanick, and M. Hoover, "Classification of Sea Ice Types With Single-
Band (33.6 GHz) Airborne Passive Microwave Imagery," J. of Geophys. Res., vol. 91, no. C9, pp. 10,611-10,694, 1986.

[3] R. Kwok, E. Rignot, B. Holt, and R. Onstoth, "Identification of Sea Ice Types in Spaceborne Synthetic Aperture Radar Data," J. of Geophys. Res., vol. 97, no. C2, pp. 2391-2402, 1992.

[4] M. Wensnahan, G.A. Maykut, and T.C. Grenfell, "Passive Microwave Remote Sensing of Thin Sea Ice Using Principle Component Analysis," J. of Geophys. Res., vol. 98, no. C7, pp. 12,453-12,467, 1993.

[5] M. Drinkwater, "Satellite Microwave Radar Observations of Antarctic Sea Ice," in Analysis of SAR Data of the PoLar Oceans, C. Tsatsoulis and R. Kwok, Eds. Berlin: Springer, 1998, pp. 145-188.

[6] M. Golden, D. Borup, M. Cheney, E. Cherkaeva, M.S. Dawson, K. Ding, A.K. Fung, D. Isaacson, S.A. Johnson, A.K. Jordan, J.A. Kong, R. Kwok, S.V. Nghiem, R.G. Onstot, J.Sylvester, D.P. Winebrenner, and I.H.H. Zabel. "Inverse Electromagnetic Scattering Models for Sea Ice," IEEE Trans. on Geosci. and Rem. Sens., vol. 36, no. 5, pp. 1675-1704, 1998.

[7] D. Long, P. Hardin, and P. Whiting, "Resolution Enhancement of Spaceborne Scatterometer Data," IEEE Trans. on Geosci and Rem. Sens., vol. 31, pp. 700-715, 1993.

[8] D.G. Long and D.L. Daum, "Spatial Enhancement of SSM/I Data," IEEE Trans. Geosci. Remote Sens, vol. 36, pp. 407-417, 1997.

[9] Q. Remund and D. Long, "Sea-Ice Extent Mapping Using Ku-Band Scatterometer Data," in press J. of Geophys. Res.

[10] WJ. Krzanowski, Principles of Multivariate Analysis: A User's Perspective, Claredon Press, Oxford, England, 1988.

[11] R.O. Duda and P.E. Hart, Pattern Classification and Scene Analysis, John Wiley and Sons, New York, New York, 1973.

[12] M. Drinkwater and G. Crocker "Modelling changes in the dielectric and scattering properties of young snowcovered sea ice at GHz frequencies," J. of Glaciology, vol. 34, no. 118, pp. 274-282, 1988. 